PROCEEDINGS OF THE

AMERICAN MATHEMATICAL SOCIETY

Volume 125, Number 4, April 1997, Pages 1071-1080

S 0002-9939(97)03608-3

\title{
Q.U.P. AND PALEY-WIENER PROPERTIES OF UNIMODULAR, ESPECIALLY NILPOTENT, LIE GROUPS
}

\author{
DIDIER ARNAL AND JEAN LUDWIG
}

(Communicated by Roe Goodman)

\begin{abstract}
We give a new proof of a weak Paley-Wiener theorem for nilpotent Lie groups due to Lipsman and Rosenberg and we introduce a general notion of Q.U.P for any unimodular locally compact group.
\end{abstract}

\section{INTRODUCTION}

0.0. Let $G$ be a locally compact group equipped with left Haar measure $m$. $\widehat{G}$ will denote the dual of $G$ (i.e. a maximal set of pairwise disjoint inequivalent continuous irreducible unitary representations of $G$ ). The Fourier transform $\hat{f}$ of a $L^{1}(G)$ function $f$ is defined by:

$$
\hat{f}(\pi)=\pi(f)=\int_{G} f(x) \pi(x) d m(x), \quad \pi \in \widehat{G} .
$$

If $f, g$ are measurable functions on $G$, we define their convolution $f * g$ by

$$
f * g(x)=\int_{G} f(y) g\left(y^{-1} x\right) d m(y)
$$

whenever the integral exists.

For a function $f$ on $G$, let

$$
f^{*}(x)=\bar{f}\left(x^{-1}\right), \quad x \in G,
$$

and

$$
{ }_{x} f(y)=f\left(x^{-1} y\right), \quad f_{x}(y)=f\left(y x^{-1}\right), \quad x, y \in G .
$$

For $f$ in $L^{1}(G)$, let $A_{f}=\{x \in G ; f(x) \neq 0\}$ and $B_{f}=\{\pi \in \widehat{G} ; \hat{f}(\pi) \neq 0\}$.

In 1973, Matolcsi and Szücs [Mat,Szu] showed that if $G$ is a locally compact abelian group, then with notation as above, $m\left(A_{f}\right) \hat{m}\left(B_{f}\right)<1$ implies $f=0$ almost everywhere (here $\hat{m}$ is the Haar measure on the dual group $\widehat{G}$, normalized so that the Plancherel identity is valid). In a 1974 preprint (which was published much later), Benedicks [Ben] showed that for $f \in L^{1}\left(\mathbb{R}^{n}\right), m\left(A_{f}\right) \hat{m}\left(B_{f}\right)<\infty$ implies $f=0$ almost everywhere, and in 1977 Amrein and Berthier [Amr,Ber] reached the same conclusion using Hilbert space techniques.

Received by the editors June 12, 1995 and, in revised form, September 21, 1995.

1991 Mathematics Subject Classification. Primary 43A30.

(C)1997 American Mathematical Society 
0.1. Now let $G$ be a unimodular group of type I. Then there exists a measure $\hat{m}$ on $\widehat{G}$ such that

$$
L^{2}(G) \simeq L^{2}(\widehat{G})=\int_{\widehat{G}}^{\oplus} \operatorname{End}_{2}\left(\mathcal{H}_{\pi}\right) d \hat{m}(\pi),
$$

i.e. the Fourier transform $f \mapsto \hat{f}$ may be extended to $L^{2}(G)$ to furnish an isometric isomorphism between $L^{2}(G)$ and the space $L^{2}(\widehat{G})$ of the measurable fields $h: \pi \mapsto$ $h(\pi)$, with $h(\pi)$ in $\operatorname{End}_{2}\left(\mathcal{H}_{\pi}\right)$ and $\int_{\widehat{G}}\|h(\pi)\|_{2}^{2} d \hat{m}(\pi)<\infty$.

Here $\operatorname{End}_{2}(\mathcal{H})$ denotes the space of the Hilbert-Schmidt operators on the Hilbert space $\mathcal{H}$ and $\|T\|_{2}$ is the Hilbert-Schmidt norm

$$
\|T\|_{2}^{2}=\sum_{k}\left\|T \phi_{k}\right\|_{\mathcal{H}}^{2}, \quad T \in \operatorname{End}_{2}(\mathcal{H}),
$$

where $\left\{\phi_{k}\right\}$ is any orthonormal basis of $\mathcal{H}$.

If $E \subset \widehat{G}$ is a measurable subset, we define $\mu(E)$ according to Hogan (see [Hog]) by

$$
\mu(E)=\int_{E}\left(\operatorname{dim} \mathcal{H}_{\pi}\right) d \hat{m}(\pi)
$$

In [Hog], a unimodular locally compact group $G$ of type I is said to satisfy the qualitative uncertainty principle (Q.U.P.) if, for each $f \in L^{2}(G)$,

$$
m\left(A_{f}\right)<m(G) \text { and } \mu\left(B_{f}\right)<\mu(\widehat{G}) \Rightarrow f=0 \quad m \text {-a.e. }
$$

It is shown in [Hog], Theorem 2.2, that any unimodular type I group with noncompact identity component satisfies the Q.U.P.

0.2. The condition $\mu\left(B_{f}\right)<\infty$ implies that for almost all $\pi \in B_{f}, \operatorname{dim}\left(\mathcal{H}_{\pi}\right)<\infty$, which is rather restrictive, and that

$$
\int_{\widehat{G}} \operatorname{rank}(\pi(f)) d \hat{m}(\pi)<\infty
$$

where $\operatorname{rank}(\pi(f))=\operatorname{dim}(\operatorname{image}(\pi(f)))$.

In this paper, we replace the condition $\mu\left(B_{f}\right)<\infty$ by the condition $(0.2 .1)$. More precisely, we define for $f$ in $L^{2}(G) \cap L^{1}(G)$ the number $\hat{\nu}(f)$ by

$$
\hat{\nu}(f)=\int_{\widehat{G}} \operatorname{rank}(\pi(f)) d \hat{m}(\pi),
$$

and we say that a locally compact unimodular type I group $G$ satisfies (Q.U.P.) if for any function $f \in L^{2}(G)$,

$$
m\left(A_{f}\right)<m(G) \text { and } \hat{\nu}(f)<\infty \Rightarrow f=0 \quad m \text {-a.e. }
$$

There is a third less restrictive candidate in the literature for the second inequality in the definition of Q.U.P., namely

$$
\hat{m}\left(B_{f}\right)<\infty
$$

But this last definition leads to very difficult still unsolved problems (see $§ 1.6$ ). 
0.3. In the first section, we prove that the property $\hat{\nu}(f)<\infty$ is equivalent to the existence of an $h \in L^{2}(G)$ such that $h=h^{*}$ and $h * f=f$ (Proposition 1.1). In fact, for a unimodular group $G$ "many" functions $f$ in $L^{2}(G)$ with the property $\hat{\nu}(f)<\infty$ do exist (Theorem 1.4). We show then that any unimodular type I group $G$, which contains a closed noncompact connected subset satisfies (Q.U.P.) (see Theorem 1.3). The proof of this theorem is of course an adaptation of the proofs in $[\mathrm{Amr}, \mathrm{Ber}]$ (as in $[\mathrm{Hog}]$ ).

We conclude section 1 with another type of weak Q.U.P. property for some particular group (Theorem 1.8).

0.4. We say that a locally compact unimodular type I group $G$ has the weak Paley-Wiener property, if any distribution $T$ on $G$ with compact support, whose Fourier transform vanishes on a subset of positive Plancherel measure, must be 0 .

Recently, Ronald L. Lipsman and Jonathan Rosenberg have shown that nilpotent Lie groups have the weak Paley-Wiener property (see [Lip,Ros]). We shall present in our second section a different proof of this result, based on the notion of adapted Fourier transform $(\S 2.2)$.

\section{Q.U.P. FOR UNIMODULAR GROUPS}

\section{a. A general Q.U.P.}

1.1. Proposition. Let $G$ be a locally compact unimodular group of type I. Let $f$ be in $L^{2}(G) \cap L^{1}(G)$. Then the number $\hat{\nu}(f)=\int_{\widehat{G}} \operatorname{rank}(\pi(f)) d \hat{m}(\pi)$ is finite if and only if there exists $h=h^{*}$ in $L^{2}(G)$, such that $h * f=f$.

Proof. Suppose that $\hat{\nu}(f)$ is finite. Then $\operatorname{rank}(\pi(f))$ must be finite for almost all $\pi$. Hence image $\left(\pi\left(f * f^{*}\right)\right)=\operatorname{image}(\pi(f))$, $\pi$-a.e. in $\widehat{G}$. Let $h_{0}=f * f^{*}$ and for $k$ in $\mathbb{N}$, let $h_{k}=\left(h_{k-1}\right)^{1 / 2}$, where we consider $h_{k}$ as an element of the $C^{*}$-algebra $C^{*}(G)$ of $G$. The limit $h=\lim _{k \rightarrow \infty} h_{k}$ exists in the von Neumann algebra $\operatorname{Vn}(G)$ of $G$ and its Fourier transform $\hat{h}$ is given for almost all $\pi$ in $\widehat{G}$ by

$$
\hat{h}(\pi)=\lim _{k \rightarrow \infty} \hat{h}_{k}(\pi)=\lim _{k \rightarrow \infty}\left(\pi\left(h_{k-1}\right)\right)^{1 / 2}=H(\pi),
$$

where $H(\pi)$ is the orthogonal projection onto the image of $\pi(f)$.

The field of operators $H: \pi \mapsto H(\pi) \in \operatorname{End}\left(\mathcal{H}_{\pi}\right)$ is measurable and is of course contained in the von Neumann algebra of the decomposable operators on $L^{2}(\widehat{G})$ (see $[\operatorname{Dix}(1)], 18.8)$. Furthermore, since

$$
\int_{\widehat{G}} \operatorname{tr}\left(H(\pi)^{2}\right) d \hat{m}(\pi)=\int_{\widehat{G}} \operatorname{rank}(\pi(f)) d \hat{m}(\pi)<\infty,
$$

we see that $H$ belongs to $L^{2}(\widehat{G})$ and so $h$ to $L^{2}(G)$. Since $H(\pi) \circ \pi(f)=\pi(f)$ for almost all $\pi \in \widehat{G}$, we have that $h * f=f$.

Conversely, suppose that $h * f=f$, for some $h=h^{*}$ in $L^{2}(G)$. Then since $\hat{h}(\pi) \circ \hat{f}(\pi)=\pi(f)$ for almost all $\pi$ in $\widehat{G}$, it follows that image $(\pi(f))$ is contained in the eigenspace of $\hat{h}(\pi)$ for the eigenvalue 1 . Hence $\operatorname{rank}(\pi(f)) \leq \operatorname{tr}\left(\hat{h}(\pi)^{2}\right)$ and

$$
\hat{\nu}(f)=\int_{\widehat{G}} \operatorname{rank}(\pi(f)) d \hat{m}(\pi) \leq \int_{\widehat{G}} \operatorname{tr}\left(\hat{h}(\pi)^{2}\right) d \hat{m}(\pi)=\|h\|_{2}^{2}<\infty .
$$

The following lemma can also be found in a slightly different form in [Hog]. 
1.2. Lemma. Let $G$ be a unimodular locally compact group which contains a closed noncompact connected subset. Let $V \subset W$ be two measurable subsets of $G$, such that $0 \neq m(V), m(W)<\infty$. Then for any $\varepsilon>0$, there exists $x \in G$, such that

$$
m(V)-2 \varepsilon<m(V \cdot x \cap W)<m(V)-\varepsilon .
$$

Proof. Clearly the connected component of the identity $G_{0}$ is not compact. Denoting the characteristic function of a subset $A$ of $G$ by $1_{A}$, we have for $x$ in $G$,

$$
m(V \cdot x \cap W)=\int_{G} 1_{V \cdot x}(y) 1_{W}(y) d m(y)=\left(1_{V}\right)^{*} * 1_{W}(x) .
$$

The functions $\left(1_{V}\right)^{*}$ and $1_{W}$ are in $L^{2}(G)$ and so $\phi=\left(1_{V}\right)^{*} * 1_{W}$ is a continuous function which vanishes at infinity. This tells us, that $\lim _{G_{0} \ni x \rightarrow \infty} \phi(x)=0$. Since $G_{0}$ is connected, the image of the restriction of $\phi$ to $G_{0}$ contains the connected subset $] 0, m(V)[$ of $\mathcal{R}$.

1.3. Theorem. Let $G$ be a locally compact unimodular group containing a closed noncompact connected subset. Let $f$ be in $L^{2}(G)$ such that $m\left(A_{f}\right)<m(G)$ and such that there exists $h$ in $L^{2}(G)$ with $h * f=f$. Then $f=0$ m-a.e.

Proof. Suppose that $f \neq 0$. We observe that for any $x \in G, h * f_{x}=f_{x}$.

Now let $C=\operatorname{supp} f=A_{f}$ and let $x_{0}=e$, the identity element of $G$. Using Lemma 1.2 we can easily find a sequence $x_{0}, x_{1}, \ldots$, in $G$, such that

$$
m(C)-\frac{1}{2^{k-1}}<m\left(C \cdot x_{k} \cap C_{k-1}\right)<m(C)-\frac{1}{2^{k}}, \quad k \in \mathbb{N},
$$

where for $k \geq 1, C_{k-1}=\bigcup_{i=0}^{k-1} C \cdot x_{i} \supset C$.

Let $C_{\infty}=\bigcup_{k=0}^{\infty} C_{k}$. We have that

$$
\begin{aligned}
m\left(C_{k+1}\right) & =m\left(C_{k} \cup C \cdot x_{k+1}\right)=m\left(C_{k}\right)+m\left(C \cdot x_{k+1} \backslash C_{k}\right) \\
& =m\left(C_{k}\right)+m\left(C \cdot x_{k+1}\right)-m\left(C \cdot x_{k+1} \cap C_{k}\right) \leq m\left(C_{k}\right)+\frac{1}{2^{k}}
\end{aligned}
$$

by (1.3.1) and the identity $m(C \cdot x)=m(C)$ for all $x$ in $G$. Hence, $m\left(C_{k+1}\right) \leq$ $m(C)+\sum_{i=0}^{k} \frac{1}{2^{i}}$, for all $k$ in $\mathbb{N}$ and so $m\left(C_{\infty}\right)<\infty$.

Let $\phi=1_{C_{\infty}}$ and consider the operator $K: L^{2}(G) \rightarrow L^{2}(G), g \mapsto \phi \cdot h * g$. Since

$$
K(g)(x)=\phi(x) \int_{G} h(y) g\left(y^{-1} x\right) d m(y)=\int_{G} \phi(x) h\left(x y^{-1}\right) g(y) d m(y),
$$

$K$ is a kernel operator with kernel function: $(x, y) \mapsto \phi(x) h\left(x y^{-1}\right)$. But this kernel function is in $L^{2}(G \times G)$ and thus $K$ is a Hilbert-Schmidt operator and hence is compact.

We have that $\operatorname{supp} f_{x_{k}}=C \cdot x_{k} \subset C_{\infty}$. Whence the functions $f_{x_{k}}$ are all eigenvectors of $K$ for the eigenvalue 1. On the other hand, again by (1.3.1),

$$
m\left(\operatorname{supp} f_{x_{k}} \backslash \bigcup_{i=0}^{k-1} \operatorname{supp} f_{x_{i}}\right) \neq 0 .
$$

The functions $f_{x_{j}}$ are thus all linearly independent in $L^{2}(G)$ and so the compact operator $K$ has an eigenspace of infinite dimension, which is impossible. We see that $f$ must be the zero function in $L^{2}(G)$. 
1.4. If $G$ is unimodular, it is easy to construct by the method of [Dix $(2)]$ elements $h, f$ in $L^{2}(G)$ such that $h \neq 0$ and $h * f=f$.

1.4. Theorem. Let $G$ be a locally compact unimodular group. The functions $f$ in $L^{2}(G)$ such that there exists $h=h^{*}$ in $L^{2}(G)$ so that $h * f=f$ are dense in $L^{2}(G)$.

Proof. Indeed, let $g=g^{*}$ in $L^{1}(G) \cap L^{2}(G)$. Define for $\lambda \in \mathbb{R}, e(i \lambda g)$ by

$$
e(i \lambda g)=i \lambda g+\frac{1}{2}(i \lambda g)^{2 *}+\frac{1}{3 !}(i \lambda g)^{3 *}+\cdots=\sum_{j=1}^{\infty} \frac{1}{j !}\left(i \lambda L_{g}\right)^{j-1}(i \lambda g)
$$

where $L_{g}$ denotes the selfadjoint operator $L_{g}(k)=g * k$ on $L^{2}(G)$. Let $q(\lambda)$ be the function $\frac{e^{i \lambda}-1}{i \lambda}$, for $\lambda$ in $\mathbb{R}$. Then the operator $\sum_{j=1}^{\infty} \frac{1}{j !}\left(i \lambda L_{g}\right)^{j-1}$ is $q\left\{L_{g}\right\}$ in the sense of functional calculus and its norm $\left\|\sum_{j=1}^{\infty} \frac{1}{j !}\left(i \lambda L_{g}\right)^{j-1}\right\|_{\text {op }}$ is less than $\|q\|_{\infty}$. Whence:

$$
e(i \lambda g) \in L^{2}(G) \quad \text { and } \quad\|e(i \lambda g)\|_{2} \leq\|g\|_{2}\|q\|_{\infty}(1+|\lambda|), \quad \forall \lambda \in \mathbb{R} .
$$

For any function $\phi$ in $C^{3}(\mathbb{R})$, with compact support, such that $\phi(0)=0$, we have $|\hat{\phi}(\lambda)|\|e(i \lambda g)\|_{2} \leq C(1+|\lambda|)^{-2}$ for some contant $C$ independent of $\lambda$ and so the integral

$$
\phi\{g\}=\frac{1}{2 \pi} \int_{\mathbb{R}} \hat{\phi}(\lambda) e(i \lambda g) d \lambda
$$

converges in $L^{2}(G)$. Furthermore we have

$$
\phi\{g\} * k=\phi\left\{L_{g}\right\}(k), \quad k \in L^{2}(G) .
$$

Indeed, $e(i \lambda g) * k=\left(\exp \left(L_{i \lambda g}\right)-1\right)(k)$ and $\left\|\exp \left(L_{i \lambda g}\right)\right\|_{\text {op }}=1$, for all $\lambda$ in $\mathbb{R}$, hence for any character $\mu$ of the (abelian) $C^{*}$-algebra generated by $L_{g}$, we have

$$
\mu\left(\int_{\mathbb{R}} \hat{\phi}(\lambda) e\left(L_{i \lambda g}\right) d \lambda\right)=\int_{\mathbb{R}} \hat{\phi}(\lambda) \exp \left(i \lambda \mu\left(L_{g}\right)\right) d \lambda=2 \pi \phi\left(\mu\left(L_{g}\right)\right),
$$

by Fourier's inversion formula.

Choose now $C^{3}$ functions $\varphi, \phi: \mathbb{R} \rightarrow \mathbb{R}$ such that $\varphi$ and $\phi$ vanish on a neighbourhood of 0 and such that $\phi \varphi=\varphi$. Let

$$
h=\int_{\mathbb{R}} \hat{\phi}(\lambda) \exp (i \lambda g) d \lambda, \quad f=\int_{\mathbb{R}} \hat{\varphi}(\lambda) \exp (i \lambda g) d \lambda \in L^{2}(G) .
$$

Then $h * f * k=\phi\left\{L_{g}\right\} \circ \varphi\left\{L_{g}\right\}(k)=\phi \varphi\left\{L_{g}\right\}(k)=\varphi\left\{L_{g}\right\}(k)=f * k$, for all $k$ in $L^{2}(G)$. Whence $h * f=f$. In order to prove density, let $k$ be any element in $L^{2}(G)$ and let $\varepsilon>0$. Choose $g=g^{*}$ in $L^{1}(G)$ continuous with compact support, such that $\|g\|_{1}=1$ and $\left\|g^{3 *} * k-k\right\|_{2} \leq \varepsilon$. Take a function $\varphi_{0}$ in $C^{3}(\mathbb{R})$ with $\varphi_{0}(t)=t^{3}$ in a neighbourhood of $]-1,1\left[\right.$. Then $\varphi_{0}\{g\}=g^{* 3}$. Choose $\varphi$ in $C^{3}(\mathbb{R})$ so that $\left\|\varphi-\varphi_{0}\right\|_{\infty}<\varepsilon$ and so that $\varphi$ vanishes in a neighbourhood of 0 . Then

$$
\begin{aligned}
\left\|\varphi\{g\} * k-g^{3 *} * k\right\|_{2} & =\left\|\varphi\{g\} * k-\varphi_{0}\{g\} * k\right\|_{2}=\left\|\left(\varphi\left\{L_{g}\right\}-\varphi_{0}\left\{L_{g}\right\}\right)(k)\right\|_{2} \\
& \leq\left\|\varphi\left\{L_{g}\right\}-\varphi_{0}\left\{L_{g}\right\}\right\|_{\text {op }}\|k\|_{2} \leq\left\|\varphi-\varphi_{0}\right\|_{\infty}\|k\|_{2}<\varepsilon\|k\|_{2} .
\end{aligned}
$$

Let $f=\varphi\{g\}, f$ is in $L^{2}(G)$. We then have

$\|f * k-k\|_{2} \leq\left(1+\|k\|_{2}\right) \varepsilon, \quad h * f * k=f * k, \quad$ for $h=\phi\{g\} \in L^{2}(G)$.

1.5. Corollary. Let $G$ be a unimodular group containing a closed noncompact connected subset. Let $f=f^{*}$ be in $L^{2}(G) \cap L^{1}(G)$ and let $\varphi$ be in $C^{3}(\mathbb{R})$ which is zero in a neighbourhood of 0 . If $m\left(A_{\varphi\{f\}}\right)$ is finite, then $\varphi\{f\}=0$. 
The proof is an immediate consequence of Theorem 1.3 and the proof of Theorem 1.4 .

\section{b. A weak Q.U.P. for special groups.}

1.6. Let $G$ be a connected simply connected nilpotent Lie group. The problem whether for such groups the condition $f \in L^{2}(G), m\left(A_{f}\right) \hat{m}\left(B_{f}\right)<\infty$ implies $f=0$ is still not settled, not even in low dimensions except for some special Heisenberg type groups (see [Ech,Kan,Kum]). Nevertheless, we shall close this section with a weaker result, for a relatively larger class of groups.

From now on, $G$ is a locally compact group containing a nontrivial central vector group $Z$. Every $\pi$ in $\widehat{G}$ restricts on $Z$ to a character $\chi_{\pi}$.

1.7. Definition. We say that a subset $\mathcal{B}$ of $\widehat{G}$ is bounded in $\widehat{Z}$, if there exists a bounded subset $\mathcal{B}_{0}$ in $\widehat{Z}$ such that for every $\pi$ in $\mathcal{B}, \chi_{\pi}$ is in $\mathcal{B}_{0}$.

We shall prove here the vanishing of any function $f$ in $L^{2}(G)$, such that $m\left(A_{f}\right)<$ $\infty$ and $B_{f}$ bounded in $\widehat{Z}$. This requirement on $f$ is clearly stronger than the condition $m\left(A_{f}\right) \hat{m}\left(B_{f}\right)<\infty$, thus we say that Theorem 1.8 is a weak Q.U.P. for G.

1.8. Theorem. Let $G$ be a unimodular amenable separable group containing a nontrivial central vector group $Z$. Let $f$ be in $L^{2}(G)$ such that $m\left(A_{f}\right)<\infty$ and such that $B_{f}$ is bounded in $\widehat{Z}$. Then $f=0$.

Proof. Since, for any $g$ in $L^{1}(G)$,

$$
\int_{G} g(x) d m(x)=\int_{G / Z}\left(\int_{Z} g(x z) d m_{Z}(z)\right) d m_{G / Z}(x),
$$

it follows from Fubini's theorem, that for almost every $x$ in $G$, the function $z \mapsto$ ${ }_{x} f_{\mid Z}(z):=f(x z)$ is in $L^{2}(Z)$ and $A\left({ }_{z} f_{\mid Z}\right)$ is of bounded Lebesgue measure. The fact that $B_{f}$ is bounded in $\widehat{Z}$ implies that there exists a bounded subset $\mathcal{B}_{0}$ in $\widehat{Z}$, such that $B_{f} \subset\left\{\pi \in \widehat{G} ; \chi_{\pi} \in \mathcal{B}_{0}\right\}$. Let

$$
\widehat{G}_{\mathcal{B}_{0}}:=\left\{\pi \in \widehat{G} \text {, such that } \chi_{\pi} \notin \mathcal{B}_{0}\right\} \text {. }
$$

Then $\widehat{G}_{\mathcal{B}_{0}}$ has empty intersection with $B_{f}$. Fix now $\chi$ in $\widehat{Z} \backslash \mathcal{B}_{0}$ and let $\rho=\operatorname{ind}_{Z}^{G} \chi$. Since $G$ is amenable, the kernel of $\rho$ in $C^{*}(G)$ is the intersection of all the $\operatorname{ker}_{C^{*}(G)} \pi$ for which $\chi_{\pi}=\chi$. It follows that $f$ is in $\operatorname{ker}_{C^{*}(G)} \rho$, i.e. $\rho(f)=0$. The kernel of $\rho$ in $L^{1}(G)$ is also given by

$$
\begin{aligned}
& \operatorname{ker}_{L^{1}(G)} \rho=\left\{g \in L^{1}(G) \text {; for almost every } x \in G,\right. \\
& \left.\qquad{ }_{x} g_{\mid Z} \in L^{1}(Z) \text { and }\left({ }_{x} g_{\mid Z}\right)^{\wedge}(\chi)=0\right\}
\end{aligned}
$$

(see [Lud]). Hence for almost all $x$ in $G,\left({ }_{x} f_{\left.\right|_{Z}}\right)^{\wedge}(\chi)=0$. If we choose a countable dense subset $\mathcal{S}$ in $\widehat{Z} \backslash \mathcal{B}_{0}$, then we see that there exists a null set $\mathcal{N}$ in $G$, such that

(i) for every $x \notin \mathcal{N}$, the function ${ }_{x} f_{\mid Z}$ is in $L^{2}(Z)$,

(ii) the support of ${ }_{x} f_{\mid Z}$ is of bounded Haar measure and

(iii) $\left({ }_{x} f_{\mid Z}\right)^{\wedge}(\chi)=0$ for all $\chi$ in $\mathcal{S}$, hence for all $\chi$ in $\widehat{Z} \backslash \mathcal{B}_{0}$.

Thus for any $x \notin \mathcal{N},\left({ }_{x} f_{\mid Z}\right)^{\wedge}$ has its support contained in the bounded subset $\mathcal{B}_{0}$, it is in $L^{2}(\widehat{Z})$ and its Fourier transform ${ }_{x} f_{\mid Z}$ vanishes on a subset of positive Lebesgue measure, since $m\left(A_{x} f\right)<\infty$. Whence Q.U.P. for the abelian group $Z$ tells us that ${ }_{x} f_{\mid Z}=0$, for all $x \notin \mathcal{N}$. Finally $f=0$. 


\section{A WeAK Paley-Wiener PRoperty For Nilpotent Lie groups}

2.0. Let $G$ be a nilpotent Lie group and let $T$ be a distribution on $G$ with compact support. For any $f$ in the Schwartz space $S(G)$ of the rapidly decreasing $C^{\infty}$ functions on $G$, the function $T * f$, defined by $T * f(x)=\left\langle T, l_{x}(\tilde{f})\right\rangle(x \in G)$, where $\tilde{f}(x)=f\left(x^{-1}\right)$, is also in $S(G)$ and $f \mapsto T * f$ defines a continuous endomorphism of $S(G)$. We can define for $\pi$ in $\widehat{G}$ the operator $\pi(T)$ which acts on the space $\mathcal{H}_{\pi}^{\infty}$ of the $C^{\infty}$-vectors of $\mathcal{H}_{\pi}$ in the following way.

By a result of Howe (see [Cor,Gre] 4.2.1), the convolution algebra $S(G)$ acts algebraically irreducibly on $\mathcal{H}_{\pi}^{\infty}$ and so it is easy to see that

$$
\pi(T)(\pi(f) \xi):=\pi(T * f) \xi, \quad f \in S(G), \xi \in \mathcal{H}_{\pi},
$$

is well defined and that $\pi(T)$ is even a continuous linear endomorphism of $\mathcal{H}_{\pi}^{\infty}$. We can thus define the Fourier transform $\widehat{T}$ of $T$ by setting $\widehat{T}(\pi)=\pi(T)$ in $\operatorname{End}\left(\mathcal{H}_{\pi}^{\infty}\right)$, $\pi$ in $\widehat{G}$.

Lipsman and Rosenberg ([Lip,Ros]) have recently proved a conjecture of Moss (see $[\mathrm{Mos}]$ ), which said that if $\widehat{T}$ vanishes on a subset of positive Plancherel measure, then $T=0$. Recently a new proof of this result has appeared in a preprint of $\mathrm{G}$. Garimella (see [Gar]). We shall present here another proof of this conjecture which is based on the notion of the adapted Fourier transform (see [Arn,Cor], [Arn,Gut] and $[\mathrm{Lud}, \mathrm{Zah}])$. Let us recall that Kirillov's theory allows us to identify $\widehat{G}$ with the space $\mathfrak{g}^{*} / G$ of the coadjoint orbits of $G$ on the dual vector space $\mathfrak{g}^{*}$ of the Lie algebra $\mathfrak{g}$ of $G$. Denote by $K$ the Kirillov mapping

$$
K: \mathfrak{g} / G \rightarrow \widehat{G}, \quad l \mapsto \pi_{l}=K\left(\operatorname{Ad}^{*}(G) l\right) .
$$

The relation between $\Omega=\operatorname{Ad}^{*}(G) l$ and $\pi=K(\Omega)$ is given by Kirillov's character formula

$$
\operatorname{tr}(\pi(f))=\int_{\Omega}(f \circ \exp )^{\wedge}(\xi) d \mu(\xi)
$$

for any $f$ in $S(G)$, where $d \mu$ denotes the $G$-invariant Kirillov measure on $\Omega$.

2.1. Pukanszky has given the following description of the orbit space $\mathfrak{g}^{*} / G$. Let $\mathfrak{B}=\left\{q_{1}, \ldots, q_{n}\right\}$ be a Jordan-Hölder basis of the $\mathfrak{g}$-module $\mathfrak{g}^{*}$. There exists a family of $r$ polynomial functions $p_{k}, 1 \leq k \leq r$, on $\mathfrak{g}^{*}$ and for every $j$ in $\{1, \ldots, r\}$ an index set $e_{j}=\left\{i_{1}(j), \ldots, i_{d(j)}(j)\right\} \subset\{1, \ldots, n\}$ with the following property:

Let $\mathfrak{g}_{j}^{*}=\left\{l \in \mathfrak{g}^{*} ; p_{1}(l)=\ldots=p_{j-1}(l)=0, p_{j}(l) \neq 0\right\}(j=1, \ldots, r)$ (here $\left.p_{0}=0\right)$. The substs $\mathfrak{g}_{j}^{*}$ form a $G$-invariant partition of $\mathfrak{g}^{*}$. There exists for every $j$ a family of functions $p_{1}^{j}, \ldots, p_{n}^{j}$ on $\mathfrak{g}_{j}^{*} \times \mathbb{R}^{d(j)}$ which are polynomials in $Z \in \mathbb{R}^{d(j)}$ with rational coefficients in $l \in \mathfrak{g}_{j}^{*}$ and the denominators of these rational functions in $l$ are powers of $p_{j}$. These $p_{i}^{j}, i=1,2, \ldots$, have the following properties:

(i) $p_{k}^{j}\left(l,\left(z_{1}, \ldots, z_{d(j)}\right)\right)$ does only depend on $l$ and on $z_{1}, \ldots, z_{k^{\prime}}$, where $i_{k^{\prime}}(j) \leq$ $k<i_{k^{\prime}+1}(j)$.

(ii) $p_{i_{k}}^{j}=z_{k}, k=1, \ldots, d(j), i_{k} \in e_{j}$.

(iii) $\operatorname{Ad}^{*}(G) l=\left\{P^{j}(l, z):=\sum_{k=0}^{n} p_{k}^{j}(l, z) q_{k}, z \in \mathbb{R}^{d(j)}\right\}, l \in \mathfrak{g}_{j}^{*}$.

Now let $d=d(1), e=e_{1}=\left\{i_{1}, \ldots, i_{d}\right\}$ and let $\mathfrak{V}=\sum_{i \notin e} \mathbb{R} q_{i}$ and $\mathfrak{V}_{1}=\mathfrak{V} \cap \mathfrak{g}_{1}^{*}$. Then for any $l \in \mathfrak{V}_{1}$ we have $l=P^{1}(l, 0)$ and the mapping

$$
\mathfrak{V}_{1} \rightarrow \mathfrak{g}_{1}^{*} / G, \quad l \mapsto \operatorname{Ad}^{*}(G) P^{1}(l, 0)
$$


is a bijection. In particular $\mathfrak{V}_{1}$ is Zariski-open in $\mathfrak{V}, \widehat{G}_{1}:=K\left(\mathfrak{g}_{1}^{*} / G\right) \subset \widehat{G}$ carries the Plancherel measure and there exists a polynomial function $Q$ on $\mathfrak{V}$ such that the measure $|Q| d l$ corresponds to the Plancherel measure on $\widehat{G}$ under the bijection $\mathfrak{V}_{1} \rightarrow \widehat{G}_{1}: l \mapsto \pi_{l}$. In fact for any measurable function $\phi$ on $\mathfrak{g}^{*}$, we have

$$
\int_{\mathfrak{g}^{*}} \phi(l) d l=\int_{\mathfrak{V}_{1}}\left(\int_{\mathbb{R}^{d(1)}} \phi\left(P^{1}(l, Z)\right) d Z\right)|Q(l)| d l .
$$

2.2. Let us now define the adapted Fourier transform (see [Lud,Zah]).

There exists a $G$-invariant Zariski-open subset $\mathfrak{g}_{0}^{*}$ of $\mathfrak{g}_{1}^{*}$ and a continuous function

$$
a: G \times \mathfrak{g}_{0}^{*} \rightarrow \mathbb{R}, \quad(x, l) \mapsto a(x, l)=\sum_{\alpha} a_{\alpha}(l) x^{\alpha}
$$

which is polynomial in $x \in G$ with rational coefficients $a_{\alpha}=\frac{Q_{\alpha}}{Q_{0}}$, whose common denominator $Q_{0}$ does not vanish on $\mathfrak{g}_{0}^{*}$ and which replaces the usual bracket $\langle l, x\rangle$ on $\mathfrak{g}^{*} \times \mathfrak{g}$, i.e., if we define the adapted Fourier transform $\hat{f}^{a}$ of an $L^{1}(G)$-function $f$ by

$$
\hat{f}^{a}(l)=\int_{G} f(x) e^{-i a(x, l)} d m(x), \quad l \in \mathfrak{g}_{0}^{*},
$$

then $\hat{f}^{a}$ is a continuous bounded function on $\mathfrak{g}_{0}^{*}$ and, for any $\pi=K\left(\operatorname{Ad}^{*}(G) l\right)$ in $\widehat{G}_{0}=K\left(\mathfrak{g}_{0}^{*} / G\right)$, for any $C^{\infty}$-function $f$ with compact support, we have

$$
\operatorname{tr}\left(\pi(f) \circ \pi(f)^{*}\right)=\int_{\operatorname{Ad}^{*}(G) l}\left|\hat{f}^{a}(\xi)\right|^{2} d \mu(\xi)
$$

where $d \mu$ is as in (2.0.1). Formula (2.2.1) allows us to deduce from $\hat{f}^{a}=0$ on $\operatorname{Ad}^{*}(G) l$ that $\pi(f)=0$, whereas $\hat{f}\left(\operatorname{Ad}^{*}(G) l\right)=0$ generally does not imply the vanishing of $\pi(f)$ (see [Lud]).

2.3. We shall give now a new proof of theorem 2.16 in [Lip,Ros]. We need first a technical result on analytic functions, for which we could not find a reference.

2.3 Lemma. Let $\mathcal{U}$ be an open connected subset of $\mathbb{R}^{n}$, let $f: \mathcal{U} \rightarrow \mathbb{C}$ be an analytic function. If $f$ vanishes on a subset of positive Lebesgue measure, then $f=0$.

Proof. (Induction on $n$ ) The lemma is of course trivial for $n=1$. Suppose now $n>1$. Let $N=\{x \in \mathcal{U}, f(x)=0\}$. Choose a box $V=[a, b] \times \bar{V}$ in $\mathcal{U}$ such that $\int_{V} 1_{N}(y) d y \neq 0$. Fubini's theorem implies that $\int_{\bar{V}} 1_{N}\left(y_{1}, \bar{y}\right) d \bar{y} \neq 0$ for all $y_{1}$ in a subset $I$ of positive Lebesgue measure in $[a, b]$. For such a $y_{1}$, the analytic function $\bar{y} \mapsto f\left(y_{1}, \bar{y}\right)$ vanishes on a set of positive Lebesgue measure and so must be 0 by the induction hypothesis. Hence $f\left(y_{1}, \bar{y}\right)=0$ for any $y_{1}$ in $I$, for every $\bar{y}$ in $\bar{V}$. Since $I$ is of positive Lebesgue measure, we see that $f$ is identically zero on $V$.

2.4 Theorem. Let $G$ be a connected simply connected nilpotent Lie group. Let $T$ be a distribution on $G$ with compact support such that $\widehat{T}$ vanishes in $\widehat{G}$ on a subset of positive Plancherel measure. Then $T=0$.

Proof. Let $\mathfrak{W}^{\prime}$ be a measurable subset of positive Plancherel measure in $\widehat{G}$ on which $\widehat{T}$ vanishes. Let $\mathfrak{W}=\left\{l \in \mathfrak{V}_{1} ; \pi_{l} \in \mathfrak{W}^{\prime}\right\}$. Since the Plancherel measure is concentrated on $K\left(\mathfrak{g}_{0}^{*} / G\right)$, and since it corresponds to $|Q|$ times Lebesgue measure on $\mathfrak{V}_{1}$, we see that $\mathfrak{W}$ is of positive Lebesgue measure and so $\mathcal{W}=\operatorname{Ad}^{*}(G) \mathfrak{W}$ is of positive Lebesgue measure in $\mathfrak{g}^{*}$ by (2.1.1). 
Let $g$ be any $C^{\infty}$ function with compact support on $G$ and let $f=T * g$. The function $f$ is itself a $C^{\infty}$ function whose support is contained in $\operatorname{supp} T \cdot \operatorname{supp} g$, whence is compact. Since $\pi(f)=\pi(T * g)=\pi(T) \circ \pi(g)$, for any $\pi$ in $\widehat{G}$, we see that $\hat{f}$ vanishes on a set which contains $\mathfrak{W}^{\prime}$. If we show that $f=0$, then $\langle T, \tilde{g}\rangle=T * g(e)=0$, and so $T$ must be 0 .

In order to prove that $f=0$, we consider $\mathfrak{g}_{0}^{*} \subset \mathfrak{g}_{0 \mathbb{C}}^{*}=\left\{\xi \in \mathfrak{g}_{\mathbb{C}}^{*} ; Q_{0}(\xi) \neq 0\right\}$. It is easy to see that $\mathfrak{g}_{0 \mathbb{C}}^{*}$ is pathwise connected and so is connected. Since the support of $f$ is compact, the integral

$$
\hat{f}^{a}(\xi)=\int_{G} f(x) e^{-i a(x, \xi)} d x
$$

exists for any $\xi$ in $\mathfrak{g}_{0 \mathbb{C}}^{*}$ and the function $\hat{f}^{a}$ is even holomorphic on $\mathfrak{g}_{0 \mathbb{C}}^{*}$, indeed, for any $\xi, \eta$ in $\mathfrak{g}_{0 \mathbb{C}}^{*}$,

$$
\frac{d}{d z} \hat{f}^{a}(\xi+z \eta)_{\mid z=0}=\int_{G}\left(-i \frac{d}{d z} a(x, \xi+z \eta)_{\mid z=0}\right) f(x) e^{-i a(x, \xi)} d x
$$

does exist and is continuous in $\xi$. Since $\hat{f}$ vanishes on $\mathfrak{W}^{\prime}$, we have $\pi_{l}(f)=0$ for any $l$ in $\mathfrak{W}$ and so by (2.2.1) $\hat{f}^{a}$ is 0 on $\mathcal{W}$, which is of positive Lebesgue measure. Hence, by Lemma 2.3, the holomorphic function $\hat{f}^{a}$ is identically 0 in $\mathfrak{g}_{0 \mathbb{C}}^{*}$, since $\mathfrak{g}_{0 \mathbb{C}}^{*}$ is connected.

Thus $\hat{f}$ vanishes on $K\left(\mathfrak{g}_{0}^{*} / G\right)$ which is dense in $\widehat{G}$. The injectivity of the Fourier transform implies that $f=0$.

\section{REFERENCES}

[Amr,Ber] W. O. Amrein and A. M. Berthier, On support properties of $L^{p}$-functions and their Fourier transforms, J. Funct. Anal. 24 (1977), 258-267. MR 57:1013

[Arn,Cor] D. Arnal and J. C. Cortet, ${ }^{*}$-product in the method of orbits for nilpotent Lie groups, J. Geom. Phys. 2(2), (1985), 85-116. MR 88a:22017

[Arn,Gut] D. Arnal and S. Gutt, Décomposition de $L^{2}(G)$ et transformation de Fourier adaptée pour un groupe de Lie nilpotent, C. R. Acad. Sci. Paris, t. 306, Série I, (1988), 25-28. MR 88m:22015

[Ben] M. Benedicks, On Fourier transforms of functions supported on sets of finite Lebesgue measure, J. Math. Anal. Appl. 106 (1985), 180-183. MR 86f:43006

[Cor,Gre] L. Corwin, F. P. Greenleaf, Representations of nilpotent Lie groups and their applications Part 1: Basic theory and examples, Cambridge studies in advanced mathematics 18 (1990). MR 92b:22007

[Cow,Pri,Sit] M. Cowling, J. F. Price and A. Sitaram, A qualitative uncertainty principle for semisimple Lie groups, J. Austral. Math. Soc. (Ser. A) 35 (1988), 127-132. MR 89d:43005

[Dix(1)] J. Dixmier, Les $C^{*}$-Algèbres et leurs Représentations, Gauthier-Villars (1969). MR 39:7442

[Dix(2)] J. Dixmier, Opérateurs de rang fini dans les représentations unitaires. Publ. Math. IHES 6 (1960), 13-25. MR 25:149

[Ech,Kan,Kum] S. Echterhoff, E. Kaniuth and A. Kumar, A qualitative uncertainty principle for certain locally compact groups, Forum Math. 3 (1991) 355-369. MR 93a:43005

[Mat,Szu] T. Matolcsi and J. Szücs, Intersection des mesures spectrales conjuguées, C. R. Acad. Sci. Paris Série A. 277 (1973), 841-843. MR 48:4804

[Gar] G. Garimella, Un théorème de Paley-Wiener pour les groupes de Lie nilpotents, preprint (1995).

[Hog] J. A. Hogan, A qualitative Uncertainty Principle For Unimodular Groups of Type I, Trans. Amer. Math. Soc. 330 (1993), 587-593. MR 94b:43003

[Lip,Ros] R. L. Lipsman and J. Rosenberg, The behavior of Fourier Transforms For Nilpotent Lie groups, preprint 1994. 
[Lud] J. Ludwig, Good ideals in the group algebra of a nilpotent Lie group, Math. Z. 161 (1983), 195-210.

[Lud,Zah] J. Ludwig and H. Zahir, On the nilpotent ${ }^{*}$-Fourier Transform, Lett. Math. Phys. 30 (1994), 23-34. MR 94k:22017

[Mos] J. D. Moss, Jr., A Paley-Wiener Theorem for selected nilpotent Lie groups, J. Funct. Anal. 114 (1993), 395-411. MR 94e:22017

Département de Mathématiques, Université de Metz, Laboratoire Méthodes Mathématiques pour l'Analyse des Systèmes, URA CNRS 399, Ile du Saulcy, 57045 Metz cedex, FRANCE

E-mail address: arnal@poncelet.univ-metz.fr

E-mail address: ludwig@poncelet.univ-metz.fr 